QCD Evolution Workshop 2014

International Journal of Modern Physics: Conference Series Vol. 37 (2015) 1560061 (9 pages)

(C) The Authors

DOI: $10.1142 / \mathrm{S} 2010194515600617$

\title{
QCD Evolution of Nuclear Quark-Gluon Correlation Function
}

\author{
Hongxi Xing* \\ Theoretical Division, Los Alamos National Laboratory, \\ Mail Stop B283, Los Alamos, NM 87545, USA \\ hxing@lanl.gov \\ Zhong-Bo Kang \\ Theoretical Division, Los Alamos National Laboratory, \\ Mail Stop B283, Los Alamos, NM 87545, USA \\ zkang@lanl.gov \\ Enke Wang \\ Institute of Particle Physics \\ and Key Laboratory of Lepton and Quark Physics (MOE), \\ Central China Normal University, Wuhan 430079, China \\ wangek@iopp.ccnu.edu.cn \\ Xin-Nian Wang \\ Nuclear Science Division, \\ Lawrence Berkeley National Laboratory \\ Berkeley, California 94720, USA \\ and \\ Institute of Particle Physics and Key Laboratory \\ of Lepton and Quark Physics (MOE), \\ Central China Normal University, Wuhan 430079, China \\ xnwang@lbl.gov
}

Published 25 February 2015

\begin{abstract}
We summarize the results on the next-to-leading order (NLO) calculations of transverse momentum broadening in semi-inclusive deeply inelastic $e+A$ scattering (SIDIS) and Drell-Yan dilepton production (DY) in $p+A$ collisions. The corresponding transverse momentum weighted differential cross sections are shown to factorize at NLO. Our calculations identify the QCD evolution equation for the quark-gluon correlation function, and also confirm the universality of the associated quark-gluon correlation function in
\end{abstract}

*Speaker.

This is an Open Access article published by World Scientific Publishing Company. It is distributed under the terms of the Creative Commons Attribution 3.0 (CC-BY) License. Further distribution of this work is permitted, provided the original work is properly cited. 
SIDIS and DY. The evolution equation can be further applied to determine the QCD factorization scale and the energy dependence of the jet transport parameter $\hat{q}$.

Keywords: QCD factorization; multiple scattering; transverse momentum broadening.

PACS numbers: 12.38.Bx, 12.39.St, 24.85.+p

\section{Introduction}

There have been a lot of efforts focused on the QCD evolution of leading-twist parton distribution functions, which are very successful in interpreting the data from high energy experiments of $e+p$ and $p+p$ collisions. However, to understand the full partonic dynamics in the presence of a large nucleus, one has to go beyond the leading-twist computation and understand the nuclear multi-parton correlations, which encode the nuclear medium properties.

One of the sensitive probes of multi-parton correlation functions is transverse momentum broadening. ${ }^{1}$ It can be studied in varies processes including semiinclusive deeply inelastic $e+A$ scattering (SIDIS) [2, 3], and Drell-Yan dilepton production (DY) as well as $J / \Psi$ production in $p+A$ collisions. ${ }^{4}$ We also have access to the multi-parton correlation functions in the study of transverse momentum imbalance of two back-to-back particle production in $e+A$ and $p+A$ collisions. ${ }^{5-7}$ So far, all the efforts in extracting the multi-parton correlation functions are based on leading order ( $\mathrm{LO}$ ) perturbative QCD calculations of multiple parton interaction in the nuclear medium, where a fixed number of quark-gluon correlation function is obtained. In order to derive how these multi-parton correlation functions evolve with the probing scale, one natural way is to go to the NLO computation. At a complete NLO computation, one will encounter various divergences, which should be properly regularized to the corresponding nonperturbative functions, so that the long and short distance physics can be factorized. This is an issue of QCD factorization of multiple scattering, ${ }^{8}$ which provides a solid baseline in extracting the multi-parton correlation functions in the nuclear medium. However, so far, a complete NLO analysis for multiple scattering is still lacking. This is largely due to the complexity of QCD dynamics for multiple scattering, which involves both initial-state and final-state interactions and medium evolution.

In this contribution, we show the first complete NLO calculation of transverse momentum broadening in SIDIS ${ }^{9,10}$ and DY in $p+A$ collisions. ${ }^{11}$ In these two processes, we have either a virtual photon prepared in the initial state or observed in the final state, therefore one can always concentrate on one particular multiple scattering effect (either initial-state or final-state) to simplify the evaluation, ${ }^{12,13}$ and to make the analysis of QCD factorization for multiple scattering more clear. On the other hand, the transverse momentum broadening in these two processes have been studied experimentally at DESY, Jlab and FNAL, and will be further investigated in the ongoing experiments at RHIC and LHC, and the future planned programs at EIC. 


\section{NLO Transverse Momentum Broadening and QCD Factorization in SIDIS and DY}

Transverse momentum broadening has long been recognized as one of the excellent probes to the QCD dynamics of multiple scattering and the medium properties as characterized by the twist- 4 multi-parton correlation functions. ${ }^{2}$ In SIDIS, transfer momentum broadening is defined as the difference of the averaged transverse momentum square between $e+A$ and $e+p$ collisions,

$$
\Delta\left\langle\ell_{h T}^{2}\right\rangle=\left\langle\ell_{h T}^{2}\right\rangle_{e+A}-\left\langle\ell_{h T}^{2}\right\rangle_{e+p},
$$

where $\ell_{h T}$ is the transverse momentum of the final state observed hadron. In this process, because the virtual photon in the initial state does not have strong interaction with the nuclear medium, the transverse momentum broadening for the produced hadron comes from final-state multiple scattering only, and the dominant contribution is from the double scattering,

$$
\Delta\left\langle\ell_{h T}^{2}\right\rangle \approx \frac{d\left\langle\ell_{h T}^{2} \sigma^{D}\right\rangle}{d \mathcal{P} \mathcal{S}} / \frac{d \sigma}{d \mathcal{P S}}, \quad \frac{d\left\langle\ell_{h T}^{2} \sigma^{D}\right\rangle}{d \mathcal{P} \mathcal{S}} \equiv \int d \ell_{h T}^{2} \ell_{h T}^{2} \frac{d \sigma^{D}}{d \mathcal{P} \mathcal{S} d \ell_{h T}^{2}},
$$

where the phase space is denoted by $d \mathcal{P S}=d x_{B} d y d z_{h}$ with $x_{B}, y, z_{h}$ the standard SIDIS variables, and the superscript " $D$ " indicates the double scattering contribution. As shown in the above equation, the transverse momentum broadening is closely related to the transverse momentum $\ell_{h T}^{2}$-weighted differential cross section, which can be computed within a generalized factorization theorem at higher twists. ${ }^{14}$

At leading order (LO), the contribution is given by Fig. 1(a). The calculation is straightforward, and the final result is directly proportional to the quark-gluon correlation function $T_{q g}$,

$$
\Delta\left\langle\ell_{h T}^{2}\right\rangle=\left(\frac{4 \pi^{2} \alpha_{s} z_{h}^{2}}{N_{c}}\right) \frac{\sum_{q} e_{q}^{2} T_{q g}\left(x_{B}, 0,0\right) D_{h / q}\left(z_{h}\right)}{\sum_{q} e_{q}^{2} f_{q / A}\left(x_{B}\right) D_{h / q}\left(z_{h}\right)} .
$$

with the quark-gluon correlation function defined as follows, ${ }^{9}$

$$
\begin{aligned}
T_{q g}\left(x_{1}, x_{2}, x_{3}\right)= & \int \frac{d y^{-}}{2 \pi} e^{i x_{1} p^{+} y^{-}} \\
& \times \int \frac{d y_{1}^{-} d y_{2}^{-}}{4 \pi} e^{i x_{2} p^{+}\left(y_{1}^{-}-y_{2}^{-}\right)} e^{i x_{3} p^{+} y_{2}^{-}} \theta\left(y_{2}^{-}\right) \theta\left(y_{1}^{-}-y^{-}\right) \\
& \times\left\langle A\left|\bar{\psi}_{q}(0) \gamma^{+} F_{\sigma}^{+}\left(y_{2}^{-}\right) F^{\sigma+}\left(y_{1}^{-}\right) \psi_{q}\left(y^{-}\right)\right| A\right\rangle .
\end{aligned}
$$
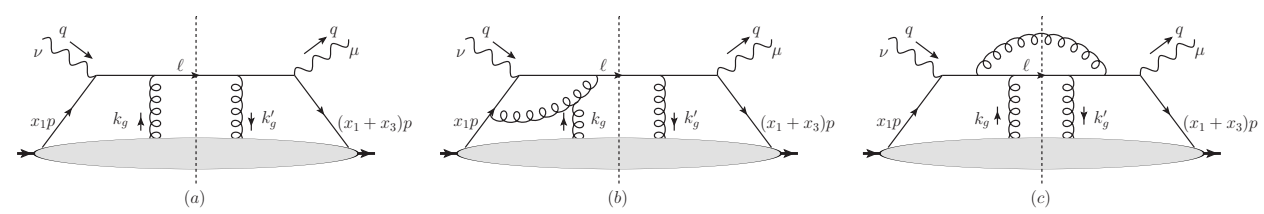

Fig. 1. Sample Feynman diagrams for double scattering contributions to the $\ell_{h T}^{2}$-weighted differential cross section from (a) leading-order (b) NLO virtual, and (c) NLO real processes. 
As in Eq. (3), the measurements of transverse momentum broadening will immediately give us the information on the twist-4 quark-gluon correlation function, and in turn the fundamental properties of the nuclear medium. However, within LO computation, what we probe is the bare quark-gluon correlation function, we are not able to investigate the scale dependences of the nonperturbative functions.

To establish a firm QCD factorization formalism for parton multiple scattering beyond LO, we will study the NLO corrections to the transverse momentum broadening. As shown in Fig. 1, the NLO contribution includes both real and virtual corrections, where the perturbative calculations of such diagrams involve divergences. We will identify these divergences and understand their physical meanings. For instance, in real diagrams as shown in Fig. 1 (c), radiative corrections reveal two types of infrared divergences from on-shell gluons. One is the so-called collinear divergence, which happens when the final-state gluon is radiated collinear to the parent quark. The other one is the so-called soft divergence, which happens when the energy of the radiated gluon approaches zero. To isolate these divergences and thus regularize them, we will work in $n=4-2 \epsilon$ dimensions with dimensional regularization. For real corrections, we study both partonic channels $\gamma^{*}+q \rightarrow q+g$ and $\gamma^{*}+g \rightarrow q+\bar{q}$, which involve quark-gluon and gluon-gluon double scattering, respectively. The calculations are rather involved, the final result contains both the divergent and the finite parts. Here we are interested in the evolution of quark-gluon correlation function, thus we list only the divergent parts, which can be expressed as follows,

$$
\begin{aligned}
& \left(\frac{4 \pi \mu^{2}}{Q^{2}}\right)^{\epsilon} \frac{1}{\Gamma(1-\epsilon)}\left\{\frac{2}{\epsilon^{2}} C_{F} \delta(1-\hat{x}) \delta(1-\hat{z}) T_{q g}(x, 0,0)-\frac{1}{\epsilon} \delta(1-\hat{x}) C_{F} \frac{1+\hat{z}^{2}}{(1-\hat{z})_{+}}\right. \\
& \quad \times T_{q g}(x, 0,0)-\frac{1}{\epsilon} \delta(1-\hat{z})\left[C_{F} \frac{1+\hat{x}^{2}}{(1-\hat{x})_{+}} T_{q g}(x, 0,0)+C_{A} \frac{2}{(1-\hat{x})_{+}}\right. \\
& \quad \times T_{q g}\left(x_{B}, x-x_{B}, 0\right)-\frac{C_{A}}{2} \frac{1+\hat{x}}{(1-\hat{x})_{+}}\left(T_{q g}\left(x, 0, x_{B}-x\right)\right. \\
& \left.\left.\left.\quad+T_{q g}\left(x_{B}, x-x_{B}, x-x_{B}\right)\right)+P_{q g}(\hat{x}) T_{g g}(x, 0,0)\right]\right\}
\end{aligned}
$$

where $\hat{x}=x_{B} / x, \hat{z}=z_{h} / z, P_{q q}$ and $P_{q g}$ are the usual quark-to-quark and gluonto-quark splitting kernels in the DGLAP evolution equations, and $T_{g g}$ is the gluongluon correlation function. ${ }^{9}$ On the other hand, the virtual correction has the following divergent part,

$$
\left(\frac{4 \pi \mu^{2}}{Q^{2}}\right)^{\epsilon} \frac{1}{\Gamma(1-\epsilon)} C_{F}\left(-\frac{2}{\epsilon^{2}}-\frac{3}{\epsilon}-8\right) \delta(1-\hat{x}) \delta(1-\hat{z}) .
$$

As one can see clearly in Eqs. (5) and (6), both real and virtual corrections contain a double pole $\left(\propto 1 / \epsilon^{2}\right)$, which represents the overlap of collinear and soft divergences. However, such double poles are canceled between real and virtual contributions, as required by collinear factorization. Thus we are left with only single 
poles $(\propto 1 / \epsilon)$, the collinear divergences. They represent the long distance physics, and should be a part of distribution or fragmentation functions. ${ }^{15}$ It is obvious that the term associated with $\delta(1-\hat{x})$ is just the collinear QCD correction to the LO quark-to-hadron fragmentation function $D_{h / q}\left(z_{h}\right)$, which should be absorbed into the definition of the renormalized quark fragmentation function,

$$
D_{h / q}\left(z_{h}, \mu_{f}^{2}\right)=D_{h / q}\left(z_{h}\right)-\frac{\alpha_{s}}{2 \pi}\left(\frac{1}{\hat{\epsilon}}+\ln \frac{\mu^{2}}{\mu_{f}^{2}}\right) \int_{z_{h}}^{1} \frac{d z}{z} P_{q q}(\hat{z}) D_{h / q}(z),
$$

where we have adopted the $\overline{\mathrm{MS}}$ scheme with $1 / \hat{\epsilon}=1 / \epsilon-\gamma_{E}+\ln (4 \pi)$, and $\mu_{f}$ is the factorization scale for the fragmentation function. The factorization scale $\mu_{f}$-dependence leads to the well-known DGLAP evolution equation for the fragmentation function $D_{h / q}\left(z_{h}, \mu_{f}^{2}\right)$.

At the same time, following the same procedure of collinear factorization, one should absorb the collinear divergence associated with $\delta(1-\hat{z})$ into the redefinition of the corresponding quark-gluon correlation function $T_{q g}\left(x_{B}, 0,0\right)$,

$$
\begin{aligned}
T_{q g}\left(x_{B}, 0,0, \mu_{f}^{2}\right)= & T_{q g}\left(x_{B}, 0,0\right)-\frac{\alpha_{s}}{2 \pi}\left(\frac{1}{\hat{\epsilon}}+\ln \frac{\mu^{2}}{\mu_{f}^{2}}\right) \\
& \times \int_{x_{B}}^{1} \frac{d x}{x}\left[\mathcal{P}_{q g \rightarrow q g} \otimes T_{q g}+P_{q g}(\hat{x}) T_{g g}(x, 0,0)\right],
\end{aligned}
$$

where $\mathcal{P}_{q g \rightarrow q g} \otimes T_{q g}$ is given by

$$
\begin{aligned}
\mathcal{P}_{q g \rightarrow q g} \otimes T_{q g} \equiv & P_{q q}(\hat{x}) T_{q g}(x, 0,0)+\frac{C_{A}}{2}\left\{\frac{4}{(1-\hat{x})_{+}} T_{q g}\left(x_{B}, x-x_{B}, 0\right)\right. \\
& \left.-\frac{1+\hat{x}}{(1-\hat{x})_{+}}\left[T_{q g}\left(x, 0, x_{B}-x\right)+T_{q g}\left(x_{B}, x-x_{B}, x-x_{B}\right)\right]\right\} .
\end{aligned}
$$

Here we have chosen the same factorization scale $\mu_{f}$ as in the fragmentation function. In principle, they do not have to be the same. After the absorption of the collinear divergences into the redefinition of nonperturbative functions, we have the QCD factorized form for the $\ell_{h T}^{2}$-weighted cross section as:

$$
\begin{aligned}
\frac{d\left\langle\ell_{h T}^{2} \sigma^{D}\right\rangle_{S I D I S}}{d \mathcal{P S}}= & \sigma_{h} \sum_{q} e_{q}^{2} \int_{x_{B}}^{1} \frac{d x}{x} \int_{z_{h}}^{1} \frac{d z}{z} D_{h / q}\left(z, \mu_{f}^{2}\right) \\
& \times\left[\delta(1-\hat{x}) \delta(1-\hat{z}) T_{q g}\left(x, 0,0, \mu_{f}^{2}\right)+\frac{\alpha_{s}}{2 \pi} H^{\mathrm{NLO}} \otimes T_{q g(g g)}\right]
\end{aligned}
$$

where $H^{\mathrm{NLO}} \otimes T_{q g(g g)}$ represent the finite NLO perturbative corrections, and have been expressed explicitly in Ref. [10].

So far, our results verify the factorization of $\ell_{h T}^{2}$-weighted differential cross section in SIDIS from multiple scattering at twist-4 in NLO. We have also verified the factorization for the transverse momentum weighted differential cross section 
of Drell-Yan lepton pair production in $p+A$ collisions at twist-4 in NLO. The perturbative result contains similar collinear divergences as that in SIDIS,

$$
\begin{aligned}
\frac{d\left\langle q_{T}^{2} \sigma^{D}\right\rangle_{D Y}}{d Q^{2}}= & \sigma_{\ell} \frac{\alpha_{s}}{2 \pi} \sum_{q} \int \frac{d x^{\prime}}{x^{\prime}} f_{\bar{q}}\left(x^{\prime}\right) \int_{x_{B}}^{1} \frac{d x}{x} \int_{0}^{1} d y\left\{\left(-\frac{1}{\hat{\epsilon}}+\ln \frac{Q^{2}}{\mu^{2}}\right)\right. \\
& \times\left[\delta(1-y) P_{q q}(z) T_{g q}(x, 0,0)+\delta(y)\left(P_{q g}(z) T_{g g}(x, 0,0)\right.\right. \\
& \left.\left.\left.+T_{g q} \otimes \mathcal{P}_{q g \rightarrow q g}\right)\right]+\cdots\right\},
\end{aligned}
$$

where the ellipses denote finite contributions, and $y=(1+\cos \theta) / 2$ with $\theta$ the partonic center-of-mass angle. Therefore, the first term in the above equation associated with $\delta(1-y)$, representing the final state gluon is radiated collinear to the projectile quark, thus we absorb it into the redefinition of beam quark distribution function, which essentially leads to the well-known DGLAP evolution equation of quark distribution function. Follow the same strategy as in SIDIS, we absorb the second term which is associated with $\delta(y)$ into the redefinition of gluon-quark correlation function. This term corresponds to the phase space where the final state gluon (quark) is radiated collinear to the parton in the nucleus. In $\overline{\mathrm{MS}}$-scheme,

$$
\begin{aligned}
T_{g q}\left(x_{B}, 0,0, \mu_{f}^{2}\right)= & T_{g q}\left(x_{B}, 0,0\right)-\frac{\alpha_{s}}{2 \pi}\left(\frac{1}{\hat{\epsilon}}+\ln \frac{\mu^{2}}{\mu_{f}^{2}}\right) \\
& \times \int_{x_{B}}^{1} \frac{d x}{x}\left[\mathcal{P}_{q g \rightarrow q g} \otimes T_{g q}+P_{q g}(z) T_{g g}\left(x_{B}, 0,0\right)\right] .
\end{aligned}
$$

We find out that the collinear correction to twist-4 quark-gluon correlation function $T_{g q}$ in DY follows exactly the same redefinition as in SIDIS in Eq. (8). This demonstrates the universality of the associated twist- 4 correlation functions and in turn implies the properties of nuclear matter as probed by a propagating parton are independent of the hard processes that produce the fast partons. After the absorption of all the collinear divergences into the corresponding nonperturbative functions, the weighted cross section in DY can be factorized into the convolution of nonperturbative functions and hard part coefficient, which is finite and free of any divergence. The finite term in DY is different from that in SIDIS, indicating that the hard part from multiple scattering are process-dependent. This confirms, for the first time, the collinear factorization for twist- 4 observables at the NLO in different processes.

\section{QCD Evolution of Quark-Gluon Correlation Function}

The quark-gluon correlation function is non-perturbative which can not be calculated by pQCD. However, its perturbative change can be derived from pQCD. From 
Eq. (8) and Eq. (12), we obtain the evolution equation for $T_{q g}$ as,

$$
\begin{aligned}
& \mu_{f}^{2} \frac{\partial}{\partial \mu_{f}^{2}} T_{q g}\left(x_{B}, 0,0, \mu_{f}^{2}\right) \\
& \quad=\frac{\alpha_{s}}{2 \pi} \int_{x_{B}}^{1} \frac{d x}{x}\left[\mathcal{P}_{q g \rightarrow q g} \otimes T_{q g}+P_{q g}(\hat{x}) T_{g g}\left(x, 0,0, \mu_{f}^{2}\right)\right] .
\end{aligned}
$$

This evolution equation essentially tells us how the quark-gluon correlation function evolves with the resolution scale of the probe. In principle, one can fix the initial condition of this evolution equation through global fitting to experimental data, and solve the evolution equation to obtain the quark-gluon correlation function for any value of resolution scale. Due to the universality of the quark-gluon correlation function, we can then apply it to any other process as long as this process involves the same quark-gluon correlation function and factorizable. This is the prediction power of $\mathrm{pQCD}$ for multiple scattering in a nuclear medium.

One of the important applications of our result is that we can further apply Eq. (13) to determine the QCD evolution of jet transport parameter $\hat{q}$. If one assumes the nucleus is loosely bound, one can neglect the correlation of nucleons inside the nucleus and relate $T_{q g}$ to the jet transport parameter $\hat{q}[16]$ :

$$
T_{q g}\left(x_{B}, 0,0, \mu_{f}^{2}\right) \approx \frac{N_{c}}{4 \pi^{2} \alpha_{\mathrm{s}}} f_{q / A}\left(x_{B}, \mu_{f}^{2}\right) \int d y^{-} \hat{q}\left(\mu_{f}^{2}, y^{-}\right) .
$$

In principle, with this relation in hand, one should be able to derive the QCD evolution of $\hat{q}$ immediately. Unfortunately, the evolution equation in Eq. (13), as it stands, is not closed (as a general feature of high-twist distributions). However, one can obtain a closed evolution equation under a certain kinematic limit. For example, in the limit of large- $x_{B}\left(x_{B} \rightarrow 1\right.$, implying also $\left.\hat{x} \rightarrow 1\right)$, the formation time for the radiated gluon, $\tau_{f}=1 /\left[x(1-\hat{x}) p^{+}\right]$, becomes much larger than the nuclear size $\left(\tau_{f} \gg R_{A}\right)$. Therefore, the interference between soft and hard rescatterings gives rise to a destructive effect to the final contribution. This effect is the socalled Landau-Pomeranchuk-Migdal (LPM) [17] interference effect which suppresses gluon radiation with large formation time. In this particular kinematic region, the medium effect from gluon rescattering disappears. Mathematically, this is manifested as the fact that the splitting kernel $\mathcal{P}_{q g \rightarrow q g} \otimes T_{q g}$ is reduced to the vacuum one $P_{q q}(\hat{x}) T_{q g}\left(x, 0,0, \mu_{f}^{2}\right)$. In other words, Eq. (13) is reduced exactly to the vacuum DGLAP evolution equation, from which we find a $\mu_{f}$-independent $\hat{q}$. Such a behavior is very similar to that of the normal parton distribution function, where the scaling behavior is observed in the large- $x_{B}$ regime.

To go beyond this large- $x_{B}$ limit, and thus study the intermediate- $x_{B}$ regime, we can expand the off-diagonal matrix elements $T_{q g}$ in Eq. (13) around $x=x_{B}$ and pick up the leading contribution. In this ansatz, we can derive a non-trivial evolution equation for $\hat{q}$. Such a evolution equation leads to not only the $\mu_{f}^{2}$-dependence, but also a $x_{B}$-dependence of $\hat{q}$. This dependence essentially tells us the violation of the scaling behavior of $\hat{q}$, again similar to that of a normal parton distribution function. 
The $x_{B}$-dependence can be also translated to the energy dependence of $\hat{q}$, as $x_{B}=$ $Q^{2} / 2 M E$ in the target rest frame. The energy dependence in the intermediate- $x_{B}$ region is consistent with early expectations. ${ }^{18}$ The implication of such an evolution equation of $\hat{q}$ will be shown in a future publication. ${ }^{19}$

\section{Conclusions}

We demonstrate through explicit calculation at NLO how QCD factorization holds for multiple parton scattering. In particular, we show for both SIDIS and DY processes that the transverse momentum weighted differential cross section can be factorized as the convolution of perturbative calculable hard part and non-perturbative functions, such as fragmentation function (parton distribution function) and twist-4 quark-gluon correlation function in SIDIS (DY). Through the calculation of transverse momentum broadening in SIDIS and DY, we verify the universality of the associated quark-gluon correlation function, which implies that the properties of nuclear matter as probed by a propagating parton are independent of the hard processes that create the fast partons. Our NLO analysis also identifies the QCD evolution equation for the twist-4 quark-gluon correlation function, which can be in turn applied to determine the QCD scale and jet energy dependence of the jet transport parameter. We further discuss the QCD evolution equation of $\hat{q}$ by looking at particular $x_{B}$-regions, where interesting scaling or scaling-violation behavior is found similar to that of normal parton distribution function.

\section{Acknowledgments}

This work is supported by U.S. DOE under Contract No. DE-AC52-06NA25396 and No. DE-AC02-05CH11231, and within the framework of the JET Collaboration, the Major State Basic Research Development Program in China (No. 2014CB845404), and the NSFC under Grants No. 11221504 and No. 10825523, and China MOST under Grant No. 2014DFG02050, and LDRD program at LANL.

\section{References}

1. J. w. Qiu and G. F. Sterman, Int. J. Mod. Phys. E 12, 149 (2003).

2. X. f. Guo, Phys. Rev. D 58, 114033 (1998).

3. X. Guo and J. w. Qiu, Phys. Rev. D 61, 096003 (2000).

4. Z. B. Kang and J. W. Qiu, Phys. Rev. D 77, 114027 (2008).

5. M. Luo, J. w. Qiu and G. F. Sterman, Phys. Rev. D 49, 4493 (1994).

6. Z. B. Kang, I. Vitev and H. Xing, Phys. Rev. D 85, 054024 (2012).

7. H. Xing, Z. B. Kang, I. Vitev and E. Wang, Phys. Rev. D 86, 094010 (2012).

8. Z. B. Kang, I. Vitev and H. Xing, Phys. Rev. Lett. 113, 062002 (2014).

9. Z.-B. Kang, E. Wang, X.-N. Wang and H. Xing, Phys. Rev. Lett. 112, 102001 (2014).

10. Z. B. Kang, E. Wang, X. N. Wang and H. Xing, arXiv:1409.1315 [hep-ph] (2014).

11. Z.-B. Kang, E. Wang, X.-N. Wang and H. Xing, in preparation.

12. X.-N. Wang and X.-f. Guo, Nucl. Phys. A 696, 788 (2001); X.-f. Guo and X.-N. Wang, Phys. Rev. Lett. 85, 3591 (2000). 
13. H. Xing, Y. Guo, E. Wang and X.-N. Wang, Nucl. Phys. A 879, 77 (2012).

14. M. Luo, J.-W. Qiu and G. F. Sterman, Phys. Rev. D 50, 1951 (1994).

15. Z.-B. Kang and J.-W. Qiu, J. Phys. G 34, S607 (2007).

16. J. Osborne and X.-N. Wang, Nucl. Phys. A 710, 281 (2002).

17. L. D. Landau and I. J. Pomeranchuk, Dolk. Akad. Nauk. SSSR 92, 92 (1953);

A. B. Migdal, Phys. Rev. 103, 1811 (1956).

18. J. Casalderrey-Solana and X.-N. Wang, Phys. Rev. C 77, 024902 (2008).

19. Z.-B. Kang, E. Wang, X.-N. Wang and H. Xing, in preparation. 